\title{
Stimulus Selectivity in Dorsal and Ventral Prefrontal Cortex after Training in Working Memory Tasks
}

\author{
Travis Meyer, ${ }^{1,2}$ Xue-Lian Qi, ${ }^{1}$ Terrence R. Stanford, ${ }^{1}$ and Christos Constantinidis ${ }^{1}$ \\ ${ }^{1}$ Department of Neurobiology and Anatomy, Wake Forest University School of Medicine, Winston-Salem, North Carolina 27157 and ${ }^{2}$ Center for the Neural \\ Basis of Cognition, Carnegie Mellon University, Pittsburgh, Pennsylvania 15213
}

The prefrontal cortex is known to represent different types of information in working memory. Contrasting theories propose that the dorsal and ventral regions of the lateral prefrontal cortex are innately specialized for the representation of spatial and nonspatial information, respectively (Goldman-Rakic, 1996), or that the two regions are shaped by the demands of cognitive tasks imposed on them (Miller, 2000). To resolve this issue, we recorded from neurons in the two regions, before and at multiple stages of training monkeys on visual working memory tasks. Before training, substantial functional differences were present between the two regions. Dorsal prefrontal cortex exhibited higher overall responsiveness to visual stimuli and higher selectivity for spatial information. After training, stimulus selectivity generally decreased, although dorsal prefrontal cortex retained higher spatial selectivity regardless of task performed. Ventral prefrontal cortex appeared to be affected to a greater extent by the nature of the task. Our results indicate that regional specialization for stimulus selectivity is present in the primate prefrontal cortex regardless of training. Dorsal areas of the prefrontal cortex are inherently organized to represent spatial information, and training has little influence on this spatial bias. Ventral areas are biased toward nonspatial information, although they are more influenced by training both in terms of activation and changes in stimulus selectivity.

\section{Introduction}

The primate prefrontal cortex is considered essential for intelligent behavior, including working memory, planning, and abstract thought (Miller and Cohen, 2001). The vast connections of the prefrontal cortex make it an ideal location to integrate information in memory and use it to guide goal-directed behaviors (Goldman-Rakic, 1987). Strong evidence suggests a particularly significant prefrontal involvement in working memory, the ability to maintain information online over a matter of seconds (for review, see Baddeley, 2003). Prefrontal neurons readily exhibit neural correlates of working memory in the form of sustained discharges that continue to represent properties of sensory stimuli, even when they are no longer present (Fuster, 1973; Funahashi et al., 1989; Constantinidis et al., 2001).

Despite intense research scrutiny, the nature of stimulus representation within the prefrontal cortex during the appearance of physical stimuli and during working memory remains a matter of debate. Longstanding anatomical (Cavada and Goldman-Rakic, 1989; Romanski et al., 1999) and physiological evidence (Wilson et al., 1993; Ó Scalaidhe et al., 1997) suggests a segregation of prefrontal cognitive function into a dorsal region dedicated pri-

\footnotetext{
Received Dec. 30, 2010; revised Jan. 28, 2011; accepted Feb. 22, 2011

This work was supported by National Institutes of Health Grant EY017077. We thank Frederik Joelving and Bill Vaughan for their contributions to the experiments, Chris Wyatt and Xin Zhou for help with anatomical imaging, and Ram Ramachandran and David Blake for helpful comments.

The authors declare no competing financial interests.

Correspondence should be addressed to Dr. Christos Constantinidis, Department of Neurobiology and Anatomy, Wake Forest University School of Medicine, Medical Center Boulevard, Winston-Salem, NC 27157.E-mail: cconstan@ wfubmc.edu.

DOI:10.1523/JNEUROSCI.6798-10.2011

Copyright $\odot 2011$ the authors $\quad 0270-6474 / 11 / 316266-11 \$ 15.00 / 0$
}

marily to representing spatial information and a ventral zone related to nonspatial information. This "domain-specific" model of organization parallels that of the dorsal and ventral visual streams (Ungerleider and Mishkin, 1982; Felleman and Van Essen, 1991).

On the other hand, more recent studies have reported the existence of individual neurons selective for both types of information throughout traditionally defined prefrontal domains and interpreted their incidence as the result of training on a task that explicitly required subjects to integrate spatial and nonspatial information (Rao et al., 1997; Rainer et al., 1998). These latter findings suggest a highly plastic prefrontal organization that is shaped by the particular cognitive demands imposed by a given external environment. We refer to this as the "integrative" model of prefrontal organization. The postulate that prior cognitive experience shapes prefrontal organization seems plausible considering that even areas of the primary sensory cortex have the capacity to reorganize after training (Recanzone et al., 1992, 1993; Li et al., 2004), and individual prefrontal neurons can alter their response properties during a single training session (Asaad et al., 1998). However, the impact of training on prefrontal organization and stimulus selectivity has never been examined directly.

To address this issue, we examined the spatial and shape selectivity of neurons in the dorsal and ventral prefrontal cortex before and after training in various working memory tasks. To acquire a baseline estimate, we recorded from the lateral prefrontal cortex of monkeys with no prior experimentation history while they viewed visual stimuli presented passively. Neurons were then sampled after training on a spatial working memory task and ultimately after training in a feature and a spatial-feature conjunction working memory task. In every phase of recording 


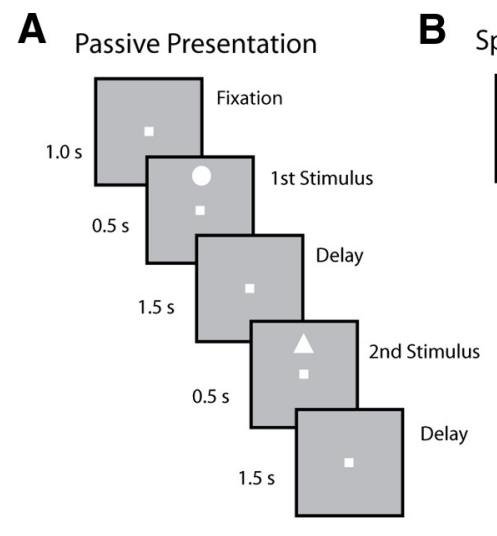

B

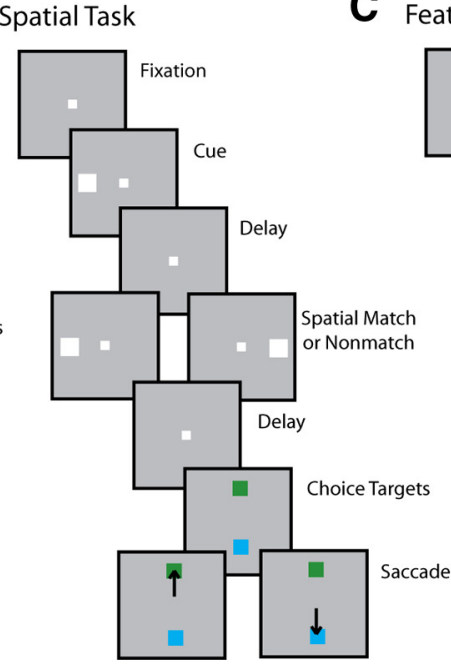

C

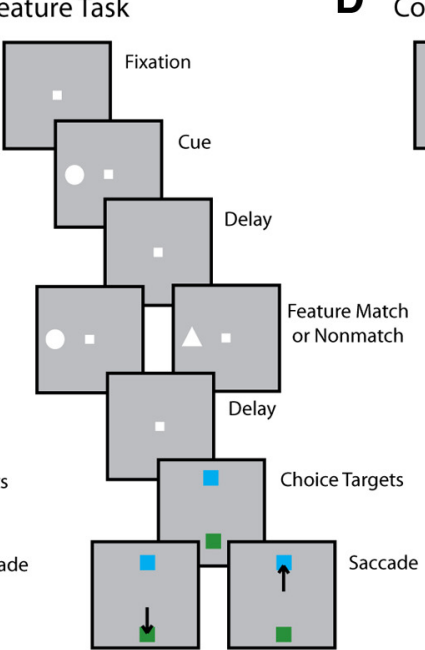

D Conjunction Task

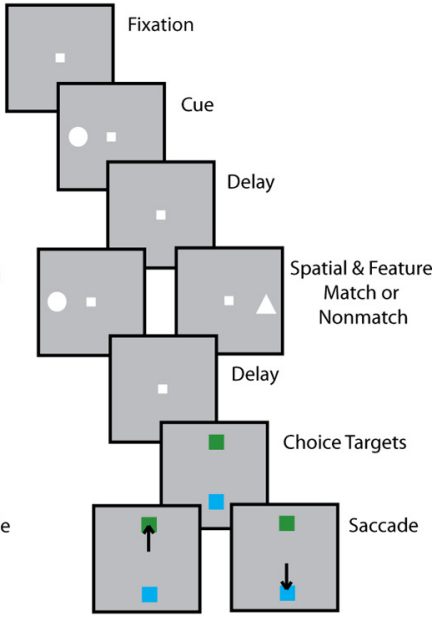

Figure 1. Behavioral tasks. Successive frames represent stimulus presentations on the screen. $\boldsymbol{A}$, Passive presentation. Stimuli of the spatial set or feature set were presented passively, while the monkey was simply required to fixate. $\boldsymbol{B}$, Spatial task. The monkey was required to remember the spatial location of the first stimulus and saccade to a green choice target if the second stimulus appeared at a matching location. C, Feature task. The monkey was required to remember the feature of a stimulus that was presented at the same location for the entire stimulus set. $\boldsymbol{D}$, Conjunction task. The monkey was required to remember both the location and feature of the cue stimulus and saccade to the green target if the second stimulus matched it in both location and feature.

we examined the properties of neurons during presentation of visual stimuli and during the delay period following it. This experimental design allowed us to test the innate organization of the prefrontal cortex and how the training regimen alters neuronal specialization.

\section{Materials and Methods}

Four male rhesus monkeys (Macaca mulatta) weighing 5-12 kg were used in these experiments. The animals had no prior experimentation experience. Neural recordings were carried out in areas 8, 9, 12, 45, and 46 of the lateral prefrontal cortex. All animal experiments were done in compliance with the guidelines set forth by the National Institutes of Health as reviewed and approved by the Wake Forest University Institutional Animal Care and Use Committee (Winston-Salem, NC).

Setup. Details of the setup have been described previously (Meyer et al., 2007). Briefly, monkeys were trained to hold their gaze on a $0.2^{\circ}$ fixation target displayed on a computer monitor. Visual stimuli were presented on the screen while eye position was monitored via an infrared eye tracking system (model RK-716; ISCAN). Eye position was sampled at $240 \mathrm{~Hz}$, digitized, and recorded. Breaks in fixation of $>2^{\circ}$ terminated the trial. Correct completion of a trial resulted in delivery of a liquid reward. Behavioral control was implemented with a custom-designed software system (Meyer and Constantinidis, 2005) that was implemented in MATLAB (MathWorks) using the Psychophysics Toolbox (Brainard, 1997; Pelli, 1997).

Stimulus presentation in pretraining phase. Stimuli were presented during the pretraining phase as described previously (Meyer et al., 2007). We used two stimulus sets, a spatial set and a feature set (see Figs. 1, 4). The spatial set involved a white $2^{\circ}$ square that appeared randomly in one of nine locations arranged on a $3 \times 3$ grid with $10^{\circ}$ spacing between stimuli. The stimulus appeared for $500 \mathrm{~ms}$ and was followed by a "delay period" that lasted for $1.5 \mathrm{~s}$ (Fig. 1A). After the delay period, a second white square appeared either in the same location or a different (typically diametric) location for $500 \mathrm{~ms}$. This was followed by a second $1.5 \mathrm{~s}$ delay period. Stimulus location was randomized from trial to trial. The feature set consisted of eight white shapes that were presented at the same location on the screen. The feature stimuli fit within a $2^{\circ}$ square and were equalized for luminance (comprised a number of pixels within $1 \%$ of each other).

During recordings, the spatial set typically was presented first, followed by the feature set. Having first assessed the spatial extent of the receptive field, feature stimuli were positioned in the spatial location that elicited the maximal response for each neuron. If no visual response was evident, stimuli were positioned over the fovea. Before obtaining any neural recordings, monkeys were familiarized with the two stimulus sets by presenting each for thousands of trials over a period of a few weeks before the beginning of experiments.

Working memory tasks. We trained the same monkeys to perform behavioral tasks that required working memory for spatial locations and features. The spatial match/non-match task (Fig. 1B) also involved presentation of two stimuli on the screen separated and followed by delay periods. After the second delay period, two choice targets appeared at locations orthogonal to the spatial stimuli. The monkey was required to saccade to the green choice target if the two stimuli matched each other in terms of spatial location and saccade to the blue choice target if the two stimuli appeared at non-matching locations. The timing of stimulus presentation was the same as that in the pretraining phase (except for the appearance of choice targets). The location of the blue and green choice targets varied randomly from trial to trial to dissociate the mnemonic component from motor planning. One monkey was trained on a different type of spatial task; this animal performed a variant of a delayed response task. The overall structure of the trial was identical to the first five frames of Figure $1 B$ except that the second stimulus was always a match and appeared at the same location as the cue. After the second delay period, no choice targets appeared and the animal was trained to saccade toward the location of the remembered visual stimulus.

In the feature match/non-match task (Fig. 1C) two stimuli were presented in succession at the same location. The monkeys were required to saccade to the green choice target if the stimuli matched in shape and to the blue choice target if they did not. As in the spatial task, the stimuli and timing were identical to the pretrained phase with the addition of two choice targets at the end. In the conjunction match/non-match task (Fig. $1 D)$ the two stimuli could differ in either spatial location or shape. The monkey was required to saccade toward the green target only if the two stimuli matched each other in both spatial location and shape. Neurons were tested with all three tasks, regardless of responsiveness or stimulus selectivity.

The selection of locations and shapes of stimuli used in recording sessions were based on the best and worst responses observed online during the stimulus set and feature set presentations. During recordings, we displayed all possible combinations of the best and worst shape and location with equal probability. Therefore, each trial had a 0.75 chance of being a non-match and a 0.25 chance of being a match. To avoid creating a bias toward non-match responses, during training we presented the animals with blocks of trials that were balanced in terms of match and non-match presentations (we included additional presentations of match 
conditions). During experiments the conjunction sets followed the spatial and feature sets, which were balanced for match and non-match stimuli. To confirm that the monkeys did not develop a biased strategy, we calculated a response bias $c$ as follows: $c=0.5 \cdot\left(Z_{\mathrm{h}}+Z_{\mathrm{f}}\right)$, where $Z_{\mathrm{h}}$ was the $z$-score corresponding to the hit rate and $\mathrm{Z}_{\mathrm{f}}$ was the $z$-score corresponding to the false alarm rate for match presentations. A response bias of +1 would mean the monkey only looked at the match target, -1 would mean that the monkey only looked at the non-match target, and 0 would reflect no bias. The two monkeys trained in the conjunction task exhibited response biases of -0.03 and -0.10 respectively. These were both near 0 , indicating that neither monkey developed a considerable response bias. Similar values were obtained for the spatial and feature sets after training (average values of -0.14 and 0.01 for the two monkeys), which were balanced in terms of match and non-match responses.

Surgery and neurophysiology. We performed surgical and neurophysiological procedures as described previously (Meyer et al., 2007). A $20 \mathrm{~mm}$ diameter craniotomy was performed over the prefrontal cortex, and a recording cylinder was implanted. The location of cylinders was assessed with anatomical magnetic resonance imaging (MRI). Neurophysiological recordings were performed with either single or multiple microelectrodes. Arrays of up to eight microelectrodes spaced $0.2-1.5 \mathrm{~mm}$ apart were advanced into the cortex through the dura with a microdrive system (EPS drive, Alpha-Omega Engineering). The electrical signal from each electrode was amplified, bandpass filtered between 500 and $8 \mathrm{kHz}$, and recorded with a modular data acquisition system (APM system, FHC, Bowdoin, ME). Waveforms that exceeded a user-defined threshold were sampled at $25 \mu$ s resolution, digitized, and stored for off-line analysis.

During experiments, electrodes were advanced into the cortex and neurons were isolated while the monkey was sitting quietly without any stimuli being displayed. After neurons were tested with all stimulus sets, a second set of neurons were sometimes collected by advancing electrodes further in the same fashion as at the beginning of the experiment. We recorded from all of the neurons that we isolated without any attempt to select neurons based on their response properties. Typically, 20 stimulus presentations were collected for each cue location or shape.

Anatomical localization. We used a grid system (Crist Instruments) to align electrode penetrations. A second recording apparatus was used in some recordings and was fitted with its own positioning system (MT micro-terminal, Alpha-Omega Engineering). The coordinates of electrode penetrations relative to the grid were recorded in each session, and a map of penetrations was generated at the end of recordings (Fig. 2). During experimental sessions, the depths of the cortex encountered by the electrodes provided a coarse map of the underlying pattern of sulci. Upon completion of the experiments, the penetration map was aligned with an image of the cortical surface obtained through anatomical MRI.

We distinguished between the two dorsal and ventral subdivisions of the lateral prefrontal cortex based on the results of anatomical studies (Cavada and Goldman-Rakic, 1989; Preuss and Goldman-Rakic, 1991). We defined the dorsolateral prefrontal cortex as the region comprising the two banks of the principal sulcus $(\leq 2 \mathrm{~mm}$ from the center of the principal sulcus), the extension of this zone posterior to the principal sulcus as far as the arcuate sulcus, and the superior convexity, dorsal to the principal sulcus. Our recordings were restricted in the anteriorposterior axis to the caudal half of the principal sulcus (see Figs. 2, 6). The region sampled incorporates the posterior aspect of area 46 and parts of areas $8 \mathrm{a}$ and 9 . We defined the ventrolateral prefrontal cortex as the cortical area in the inferior convexity of the prefrontal cortex lateral to the principal sulcus ( $>2 \mathrm{~mm}$ from the center of the principal sulcus). This region incorporates parts of areas 12 and 45 (see Figs. 2, 6). For simplicity, we henceforth refer to these two regions as "dorsal" and "ventral," respectively.

Data analysis. We separated action potential waveforms into separate units using an automated cluster analysis method based on the KlustaKwik algorithm (Harris et al., 2000), which relied on principle component analysis of the waveforms. Firing rate of units was then determined for each of the task epochs. We then identified neurons that exhibited firing rates significantly different from the firing rate recorded in the $1 \mathrm{~s}$ interval of fixation (paired $t$ test, $p<0.05$ ) for either a spatial or a shape stimulus. We also identified neurons with a significant decrease of firing rate in a
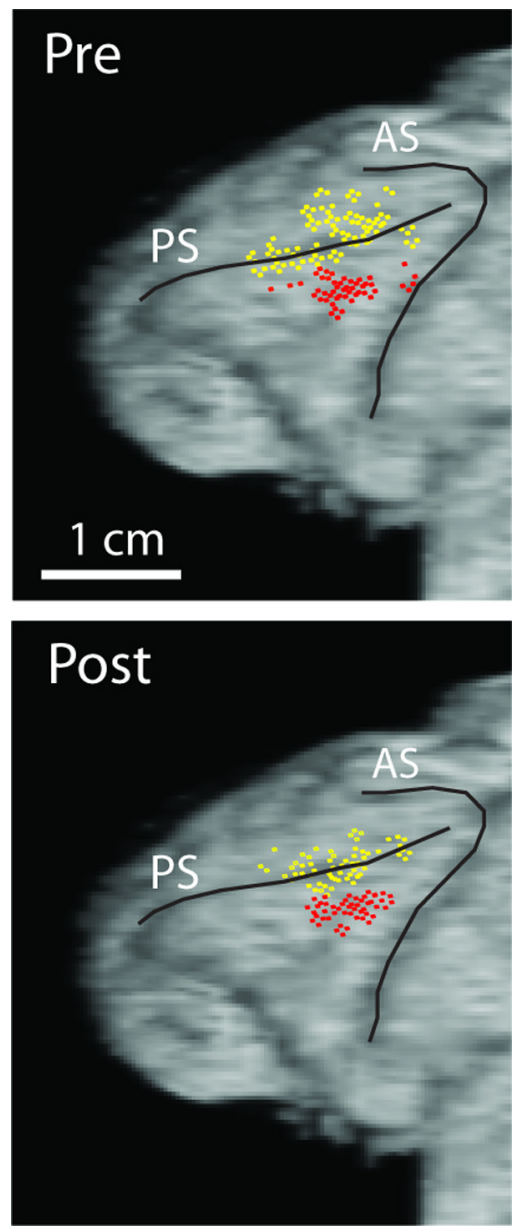

Figure 2. Lateral view of a monkey brain obtained through MRI, with electrode penetrations before and after training superimposed. Penetrations sampling the dorsal and ventral prefrontal cortex are colored in yellow and red respectively. Additional recordings were obtained from the opposite hemisphere of this animal after training. AS, Arcuate sulcus; PS, principal sulcus.

task epoch in the absence of an elevated firing rate in response to another stimulus presentation in the same task epoch. Only trials that resulted in correct behavioral responses to the task are presented in this paper.

To ensure the stability of the firing rate in the recordings analyzed, we identified recordings in which a significant effect of trial presentation sequence was evident in the baseline firing rate (ANOVA, $p<0.05$ ), e.g., because of a neuron disappearing or appearing during a run as we were collecting data from multiple electrodes. Data from these sessions were truncated so that analysis was performed on a range of trials with a stable firing rate.

The spatial selectivity of visually responsive neurons was assessed by comparing the discharge rates during the presentation of the first stimulus at the nine grid locations. Neurons with significantly different responses to the nine stimulus locations (ANOVA, $p<0.05$ ) were considered spatially selective. Likewise, neurons were considered shape selective if their responses to the eight shapes were significantly different (ANOVA, $p<0.05$ ). The best (most responsive) and worst (least responsive) locations and shapes were also determined.

The degree of spatial selectivity of each neuron was quantified using a selectivity index defined as (Max - Min)/(Max + Min), where Max and Min represent the neuron's highest (maximum) and lowest (minimum) firing rate for the nine stimuli, respectively. We used the same index to quantify shape selectivity as well.

To test the possibility that systematic differences in the selection of neurons before and after various stages of training accounted for the findings, we analyzed multiunit records created by pooling together 
spikes of all neuron waveforms extracted from each electrode (Compte et al., 2001). We then repeated the analysis of results, treating the multiunit record as a single neuron. We also duplicated the analysis for single neurons, recorded before and after training, matched for signal-to-noise ratio (SNR). For each neuron, we defined SNR as the ratio between the peak-to-trough amplitude of the mean action potential waveform divided by the standard deviation (SD) of the noise computed from the baseline of each waveform derived from the first 10 data points (corresponding to $0.25 \mathrm{~ms}$ ) of each sample. We repeated our analysis for neurons with SNR values $>6$, yielding neuronal samples matched for signal-to-noise ratios.

\section{Results}

\section{Pretraining results}

Data were collected from four monkeys that had no prior training on any laboratory task. During the initial phase of data collection, the subjects were required only to maintain fixation while stimuli were presented on a screen. A trial consisted of the sequential presentation of two visual stimuli separated by a delay interval during which only the fixation stimulus remained on the screen (Fig. 1A). Stimuli were drawn from one of two stimulus sets: a spatial set consisting of white squares presented on a $3 \times 3$ grid of $10^{\circ}$ spacing between stimuli, and a feature set composed of eight white geometric figures. A total of 1365 neurons were recorded using an unbiased sampling strategy in the ventral and dorsal subdivisions of the lateral prefrontal cortex (Fig. 2). At this stage, we sought to ascertain whether functional differences existed between the ventral and the dorsal prefrontal cortex as postulated by the domain-specific model, although such a result would not necessarily be incompatible with the integrative model. On the other hand, a finding of no significant differences between the two subdivisions at this stage would only be consistent with the integrative model.

\section{Visual responses}

Before training, significant differences between the dorsal prefrontal cortex and the ventral prefrontal cortex were readily apparent in both the incidence of neurons responsive to visual stimuli and their selectivity for visual stimulus properties. We identified neurons responding to visual stimuli based on a significantly increased firing rate during the stimulus presentation relative to baseline (paired $t$ test, $p<0.05$ ). The percentage of neurons exhibiting such visual responses was significantly higher $\left(\chi^{2}\right.$ test, $\left.p<10^{-20}\right)$ in the dorsal $(37 \%, N=287)$ than in the ventral region $(9 \%, N=55)$ of the prefrontal cortex (Fig. $3 A)$.

We evaluated the stimulus selectivity of visually responsive neurons (287 dorsal and 55 ventral) by comparing their firing rates during the presentation of the different spatial and feature stimuli. The spatial set was presented first, followed by the feature set. Typical responses of a dorsal prefrontal neuron are shown in Figure 4. The neuron was highly selective for spatial locations, responding best to a stimulus appearing directly to the right of the fixation point (Fig. 4A). The neuron was also significantly selective for shapes (Fig. $4 B$, arranged in order of decreasing responses) although to a lesser degree, as all eight stimuli elicited some response.

Overall, spatial selectivity was more strongly associated with the dorsal region, where $70 \%$ of visually responsive neurons exhibited significantly different responses across the nine stimulus locations tested (ANOVA, $p<0.05$ ). In contrast, only $25 \%$ of visually responsive neurons in the ventral regions showed spatial selectivity ( $\chi^{2}$ test, $\left.p<0.005\right)$. Furthermore, the degree of spatial selectivity of neurons responding to visual stimuli was significantly different between areas. The average selectivity index (SI),
A

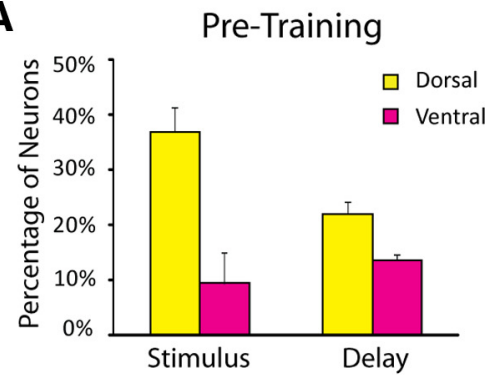

B

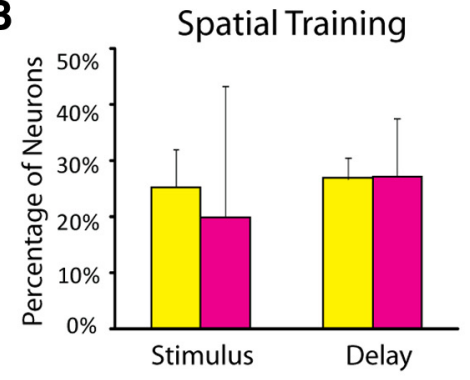

C Post-Training

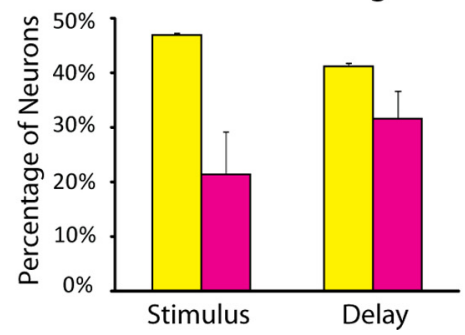

Figure 3. A, Percentage of neurons with significantly elevated firing rate during the presentation of visual stimuli and during the delay period before training in the working memory tasks. Color of bars indicates prefrontal region. Average percentages are shown for results pooled across all monkeys. Error bars denote SE of percentages observed across monkeys. $\boldsymbol{B}$, Percentage of neurons with significant stimulus and delay period responses in two monkeys trained to perform the spatial working memory task only. C, Percentage of neurons with stimulus and delay period responses in two monkeys trained to perform all three working memory tasks.

which quantified the magnitude of selectivity, indicated that dorsal neurons were more selective ( $t$ test, $p<0.05$ ) (Fig. $5 A$ ). Response latencies to the stimuli did not differ significantly between areas (data not shown).

With regard to shape selectivity, we observed no significant differences in the percentage of shape-selective neurons across regions but significant differences in terms of the magnitude of selectivity; neurons encountered in the ventral prefrontal cortex (Fig. $5 B$ ) were most selective for our feature set ( $t$ test; $p<0.05$ ). Because dorsal and ventral prefrontal neurons differed in their spatial selectivity and because shape selectivity may depend on the spatial location tested (e.g., neurons highly selective for shapes may be most sensitive in the fovea), we sought to ensure that the regional differences in shape selectivity were not in some way caused by differences in spatial properties. For this reason, we refined our analysis and processed separately two groups of neurons. First, we examined neurons with significant spatial selectivity. For those, we relied on responses obtained from stimulus presentations in the receptive field at locations where firing rates with $>50 \%$ of the maximum response were obtained during presentation of the spatial set. This analysis confirmed that shape selectivity was significantly higher for ventral than for dorsal prefrontal neurons before training ( $t$ test, $p<0.05$ ). Second, we examined neurons that did not exhibit spatial selectivity. For 
A

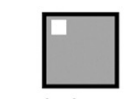

:
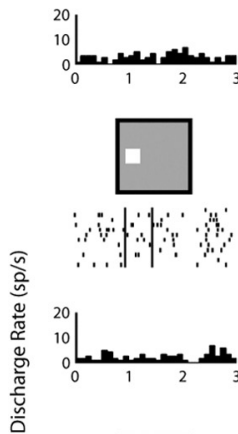

$\square$

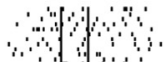

20

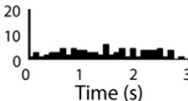

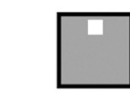

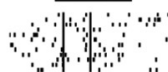

${ }^{20}$
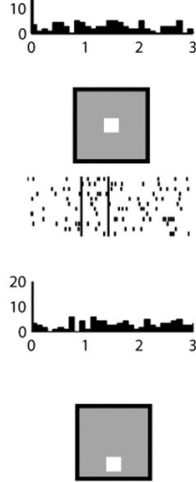

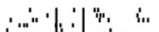

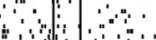

20
10

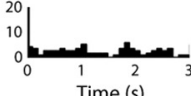

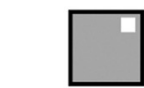

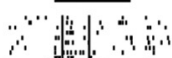

(1)
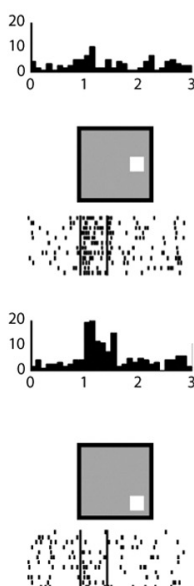

20
10

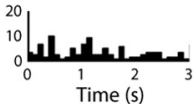

B
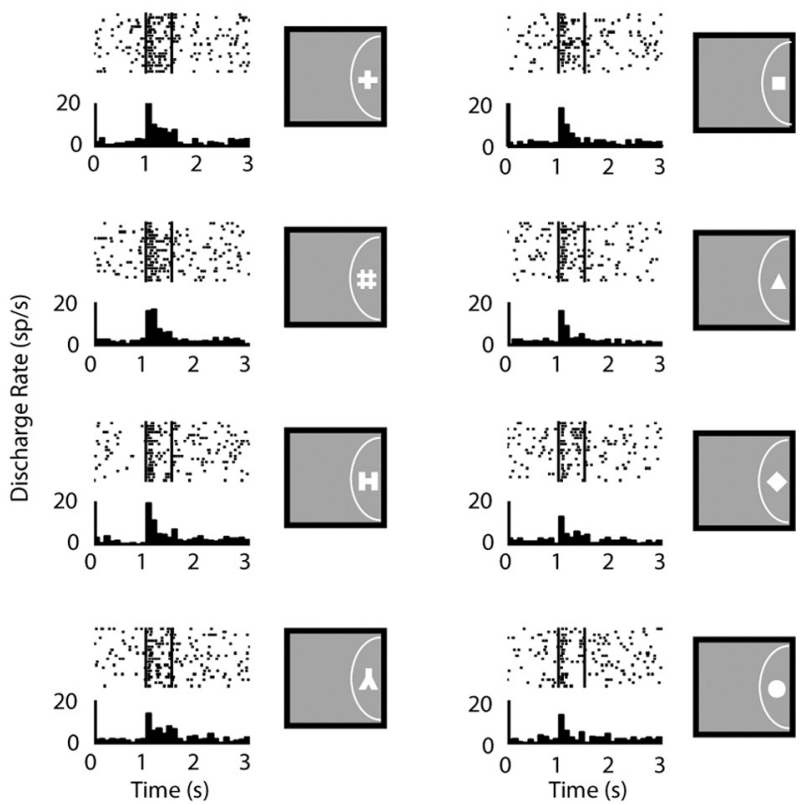

Figure 4. Rasters and peristimulus time histograms of a single neuron in the dorsal prefrontal cortex recorded before training. $\boldsymbol{A}$, Responses recorded in the spatial set. The neuron has significant spatial selectivity with maximal responses to the right of fixation. $\boldsymbol{B}$, Responses recorded in the feature set. Responses have been arranged in order of response magnitude during the stimulus presentation. The neuron had broad but statistically significant selectivity to the feature stimuli as well (ANOVA, $p<0.05)$.

these, we relied on responses obtained from presentation of the feature set at the fovea. Regional difference was also significant $(t$ test, $p<0.05)$ and even more pronounced for this group of neurons $(31 \%$ difference in mean SI values between ventral and dorsal regions vs $25 \%$ for the corresponding groups with significant spatial selectivity). The result is not surprising considering that the receptive fields of highly feature-selective neurons in the inferior temporal cortex (a major afferent of the ventral prefrontal cortex) almost always include the fovea (Gross et al., 1972, 1979; Schwartz et al., 1983; Tovee et al., 1994; Op De Beeck and Vogels, 2000).

We should emphasize that despite the overall regional specialization, segregation of spatial and shape information processing was not absolute; some dorsal prefrontal neurons exhibited shape selectivity (and ventral neurons exhibited spatial selectivity). We also encountered integrative neurons, selective for both types of stimuli (Fig. 4) in all four of the monkeys.

\section{Delay period responses}

The incidence and selectivity of neurons with delay period activity also displayed regional differences, mirroring the effects observed in the stimulus presentation period. We should note that although the monkeys in this phase of recording were not required to remember the stimuli, we did observe neurons with significantly elevated activity in the delay period and selectivity for the properties of the preceding stimulus, as described previously (Meyer et al., 2007). Dorsal prefrontal cortex contained a significantly higher percentage of neurons with delay period responses $(22 \%)$ than did ventral prefrontal cortex $(14 \%)$ ( $\chi^{2}$ test, $p<0.005)$. Among neurons with delay period activity, regional specialization was also observed with respect to spatial selectivity; $43 \%$ of dorsal neurons exhibited significant selectivity for locations (ANOVA; $p<0.05$ ) whereas only $4 \%$ of ventral neurons exhibited spatial selectivity, which constituted a significant difference ( $\chi^{2}$ test, $p<10^{-20}$ ). The magnitude of spatial selectivity was also different as evidenced by a significantly higher mean SI value for dorsal compared to ventral neurons $(0.43$ vs $0.30, t$ test, $p<0.005)$. Twenty-seven percent of dorsal and $9 \%$ of ventral neurons with significantly elevated delay period activity exhibited shape selectivity in the delay period.

\section{Spatial task training}

Once recordings in the naive state were completed, two of the monkeys ( $\mathrm{AD}$ and $\mathrm{BE}$ ) were trained on a spatial working memory task using the same stimuli they had previously viewed passively (Fig. $1 B$ ). The animals now had to remember the spatial location of the stimuli to execute the task. The animals continued to view the stimuli of the feature set passively, having not been trained to recognize or remember them at this stage. We recorded from a total of 339 neurons, using the same unbiased sampling strategy. At this stage, we sought to determine how training affects regional specialization and alters the spatial and shape selectivity of prefrontal neurons. No change in regional specialization or functional properties of neurons would be consistent with the domain-specific model, suggesting an innate and immutable regional specialization. On the other hand, an increase in the incidence and magnitude of spatial selectivity would be consistent with the integrative model, which postulates that the cognitive task performed shapes stimulus selectivity and may induce regional differences.

\section{Visual responses}

After training on a spatial working memory task, 25\% (61) of dorsal prefrontal neurons and 20\% (19) of ventral prefrontal neurons exhibited significant visual responses (Fig. 3B). This represented a significant increase in the percentage of visually responsive neurons in ventral prefrontal cortex $\left(\chi^{2}\right.$ test, $p<$ $0.005)$. The overall firing rate of visually responsive neurons increased after training (Fig. 6). Spatial selectivity in dorsal prefrontal cortex exhibited the opposite trend than that predicted by the 
A

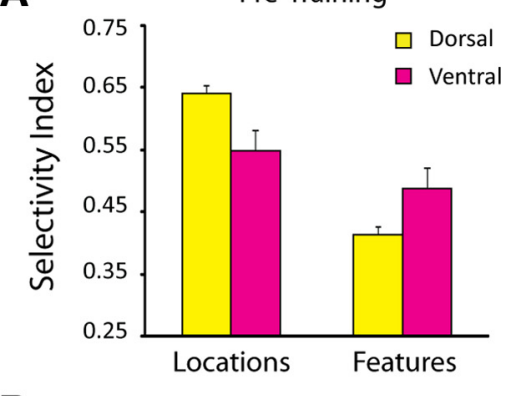

B
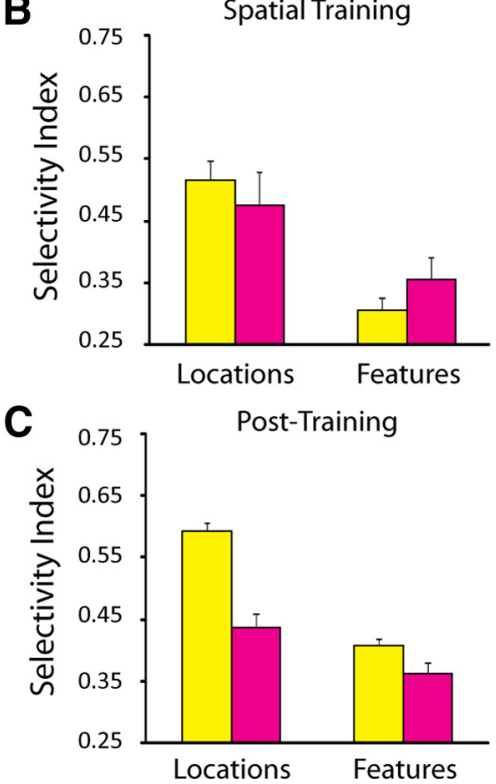

Figure 5. Average values of spatial and shape selectivity index for each region of the prefrontal cortex. Color of bars indicates prefrontal region as in Figure 3. Error bars represent SE of data averaged in each bar. $\boldsymbol{A}$, Data from monkeys before training. $\boldsymbol{B}$, Data from monkeys trained only in the spatial working memory tasks. $C$, Data from monkeys trained in spatial, feature, and conjunction tasks.

integrative model: there was a decrease in the percentage of neurons with spatially selective responses (from 70 to $47 \%$ ) that represented a change at the margin of statistical significance $\left(\chi^{2}\right.$ test, $p=0.05)$ and a decrease in the magnitude of selectivity (Fig. $5 A, B)$ as evidenced by a significant decrease in the average SI value ( $t$ test, $p<0.005)$. We observed a decrease in magnitude of shape selectivity as well (Fig. $5 A, B$ ), which was significant for both dorsal prefrontal cortex and ventral prefrontal cortex ( $t$ test, $p<0.005$ in both cases). These changes could not be accounted for by differences in selection of neurons or quality of recordings before and after training; a decrease in selectivity for both regions and stimulus types was evident when we calculated SI values based on multiunit records and on samples of neurons matched for signal-to-noise ratios (see Materials and Methods).

To gain insight into the decrease in selectivity, we examined the tuning functions of neurons before and after training in the behavioral task. Although the firing rate during spatial stimulus presentation increased after training, this was the result of a generalized increase of activity observed both for effective and ineffective stimuli (Fig. $7 A, B$ ). This decreased the contrast between the best and worst stimulus response, which defines the selectivity index. Interestingly, we observed the same decrease in selectivity for both spatial stimuli, which the monkeys were trained to remember, and feature stimuli, which continued to be presented passively (Fig. 7C,D). This finding suggested that training to perform the task induced activity changes that altered the representation of information for any stimulus. We also found that essentially the same changes took place in both the dorsal prefrontal cortex (Fig. 7A,C) and the ventral prefrontal cortex (Fig. $7 B, D$ ).

\section{Delay responses}

Recordings after training revealed an increase in the number of neurons with significantly elevated activity in the delay period (from 21 to $27 \%$ in dorsal prefrontal cortex and from 15 to $27 \%$ in ventral prefrontal cortex). The firing rate of neurons with significant responses during the stimulus presentation was also higher in the delay period for both the dorsal and the ventral prefrontal cortexes (Fig. 6). Notably, the increase in delay period activity following the presentation of a stimulus was greater for the spatial stimuli, which monkeys had to remember (Fig. 6A,B), than for the feature stimuli, which continued to be presented passively (Fig. 6C,D). Even after subtracting the baseline fixation firing rate, delay period activity following the spatial stimuli increased significantly after training $(t$ test, $p<0.05$ for both the dorsal and ventral prefrontal cortexes). On the other hand, delay period activity (over the baseline) following the feature stimuli was actually slightly lower after training in the spatial working memory task ( -0.9 and -0.4 spikes/s for the dorsal prefrontal cortex and ventral prefrontal cortex, respectively).

Changes in the percentage of neurons with significant selectivity for stimulus properties in the delay period mirrored the effects observed in the stimulus presentation period. The percentage of neurons with delay period spatial selectivity in the dorsal prefrontal cortex declined after training in the spatial working memory task ( $37 \%$ pre vs $27 \%$ post), contrary to the prediction of the integrative hypothesis. The percentage of dorsal prefrontal neurons with spatial selectivity in the delay period $(27 \%)$ continued to be higher than the equivalent percentage of ventral prefrontal neurons (17\%). Four and fifteen percent of dorsal and ventral prefrontal neurons were significantly selective for shapes during the delay period, respectively.

\section{Feature and conjunction task training}

We trained two monkeys who had previously viewed the stimuli passively (EL and $\mathrm{AD}$ ) to perform all three working memory tasks (Fig. 1): a task that required spatial working memory (Fig. $1 B$ ), a task requiring feature working memory (Fig. 1C), and a task requiring working memory for the conjunction of locations and features (Fig. 1D). The animals were first trained in the spatial working memory task and then the feature working memory task. After completing the spatial training, the monkeys showed no understanding of the feature working memory task and had to be trained to remember the features of the stimuli. Training in the conjunction task was performed last. Training required up to 1 year for mastery of all three tasks. Average performance in the behavioral sessions that contributed to the present dataset was $92 \%$ for the spatial task, $84 \%$ for the feature task, and $82 \%$ for the conjunction task.

We repeated neurophysiological recordings in the same cortical areas, in some instances revisiting the same cortical tracks, and repeated the analysis of neuronal responses. A total of 1012 neurons were recorded after training. At this stage, our primary objective was to determine whether performing a task that requires remembering both the features and location of a stimulus increases the incidence of prefrontal neurons selective for both types of information and eliminates (or diminishes) differences 


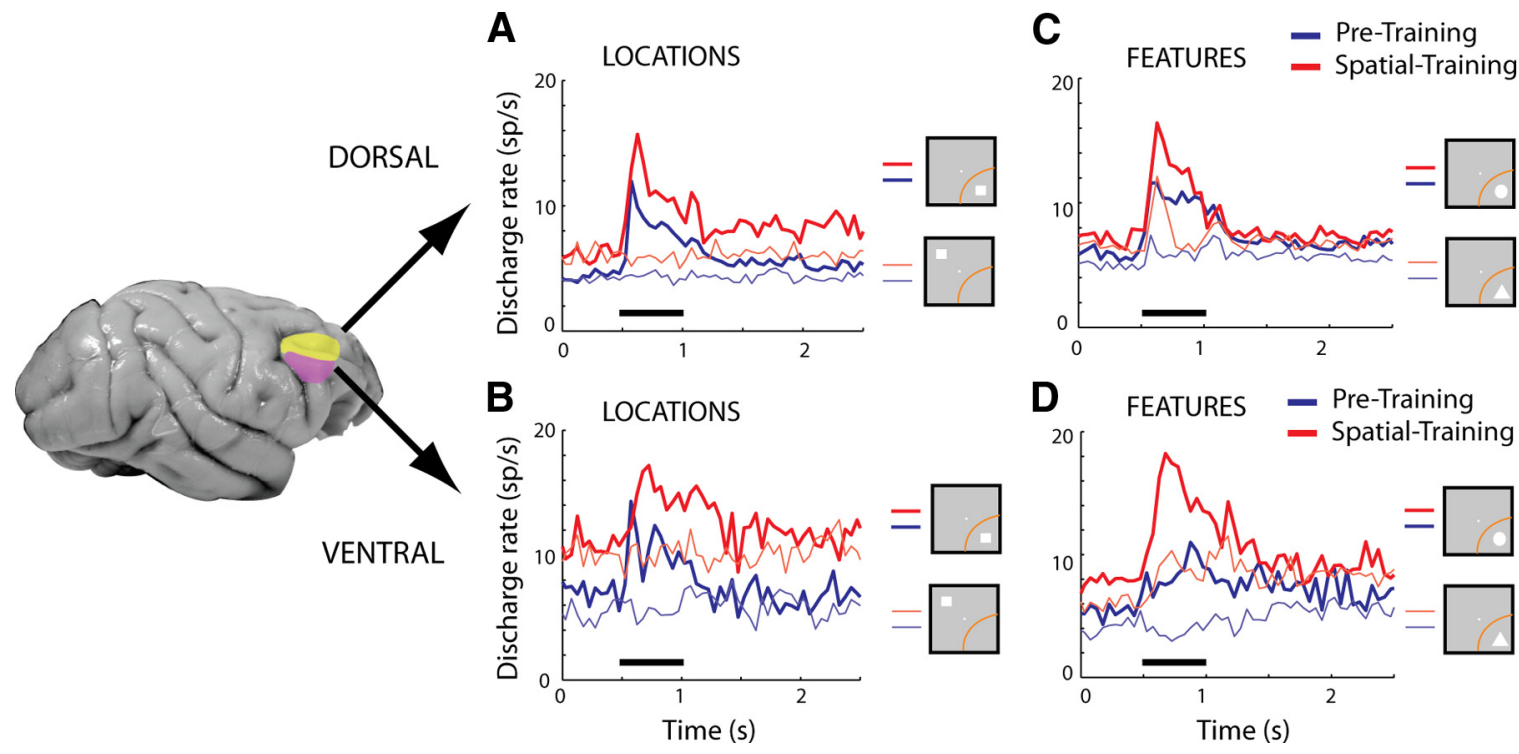

Figure 6. $\quad A, B$, Population peristimulus time histograms (PSTHs) averaging all visually responsive neurons (selective or not) recorded from each prefrontal region during presentation of spatial stimuli. Blue lines represent responses before training and red lines represent responses after training in the spatial working memory task. Insets to the right of each PSTH indicate appearance of the first stimulus in the best location in the receptive field (heavy lines) or out of the receptive field (light lines). The actual locations of the stimuli differed for each neuron. C, D, Population PSTHs of responses recorded during presentation of the feature set. Insets represent presentation of the feature stimulus that elicited the best (heavy lines) or the worst response (thin lines).

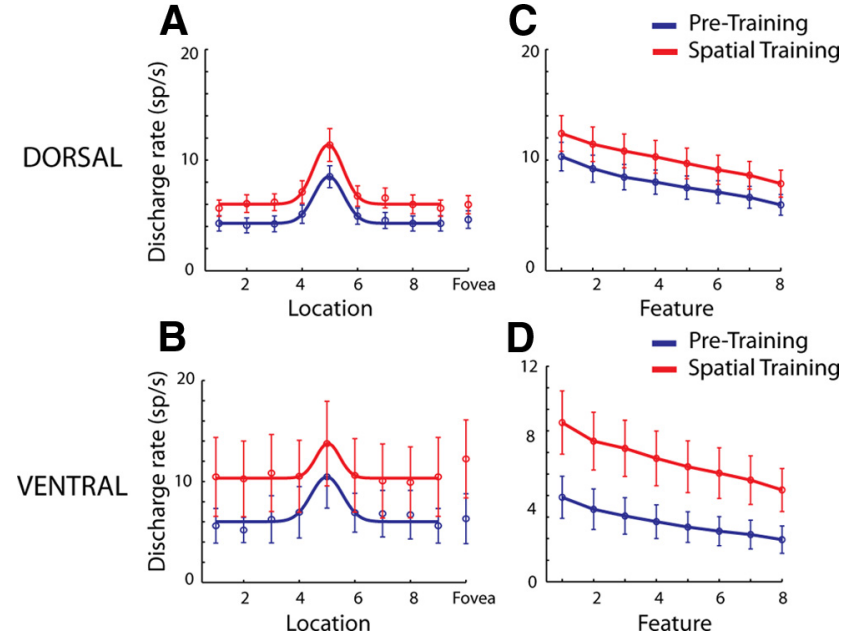

Figure 7. $A, B$, Average discharge rate of neurons responding during the presentation of spatial stimuli are plotted for each prefrontal subdivision and for before and after training in the spatial working memory task. The arrangement of spatial locations has been rotated so that the best response is at location 5 for every neuron; responses at the fovea are plotted separately. Line represents Gaussian fit. Locations 1 and 9 are the same. Error bars represent SEs. C, D, Average discharge rate during the presentation of stimuli in the feature set. Features have been rank ordered from best to worst for each neuron.

in regional specialization of the dorsal and ventral prefrontal cortex, two key tenets of the integrative model.

\section{Visual responses}

After training the monkeys in all three working memory tasks, $47 \%$ (286) of dorsal and 22\% (87) of ventral prefrontal neurons were driven by the visual stimuli (Fig. $3 C$ ). Similar to what we observed after training in the spatial task alone, firing rates during the stimulus presentation increased, particularly for the ventral prefrontal cortex (Fig. 8).

In terms of stimulus selectivity, we saw a decline in the proportion of neurons selective for spatial and shape information compared to the same proportions before any training (Fig. 9).
This was true for both the dorsal prefrontal cortex and the ventral prefrontal cortex. Still, a greater proportion of dorsal neurons than ventral neurons was spatially selective (58 to $24 \%, \chi^{2}$ test, $p<0.005)$. A reduction in proportion was also seen for neurons selective for both locations and shapes (Fig. 9), although the monkeys had learned to perform the conjunction task which required them to remember both stimulus location and shape over the delay period. The decrease of these integrative neurons reached statistical significance in the dorsal prefrontal cortex $\left(\chi^{2}\right.$ test, $p<0.05$ ). To ensure that this decrease in selectivity was not the result of damage accumulated from repeated penetrations, we conducted recordings in the dorsal prefrontal cortex in both the same ( $n=167$ neurons) and the opposite hemisphere $(n=308$ neurons) of one monkey after training. We found virtually identical percentages of neurons that were selective for the stimuli in the resampled and newly sampled hemisphere (57 and 59\% for spatial stimuli and 21 and $28 \%$ for feature stimuli, respectively). The percentage of neurons with spatial selectivity in the newly sampled hemisphere alone was significantly diminished ( $\chi^{2}$ test, $p<0.05)$ compared to the percentage of neurons with selectivity before training in this monkey $(82 \%)$. The percentage of neurons with shape selectivity was also greatly diminished compared to that before training (46\%). Repeating the analysis for multiunit records and samples of neurons matched for signal-to-noise ratio also indicated a decrease in the percentage of units selective for locations and shapes. These findings run counter to the idea that training on a conjunction task leads to increases in spatial and shape selectivity that crosses traditional domain boundaries (Rao et al., 1997).

Reduction in stimulus selectivity did not involve only the percentage of neurons; the magnitude of stimulus selectivity also decreased after training (Fig. $5 C$ ). This was true for both prefrontal subdivisions and for both types of information (two-way ANOVA, significant main effect of training, $p<0.005$ for spatial and feature information). The difference in the magnitude of spatial selectivity between dorsal prefrontal cortex and ventral prefrontal cortex remained significant ( $t$ test, $p<0.005$ ). On the other hand, the decrease in shape selectivity in dorsal prefrontal 


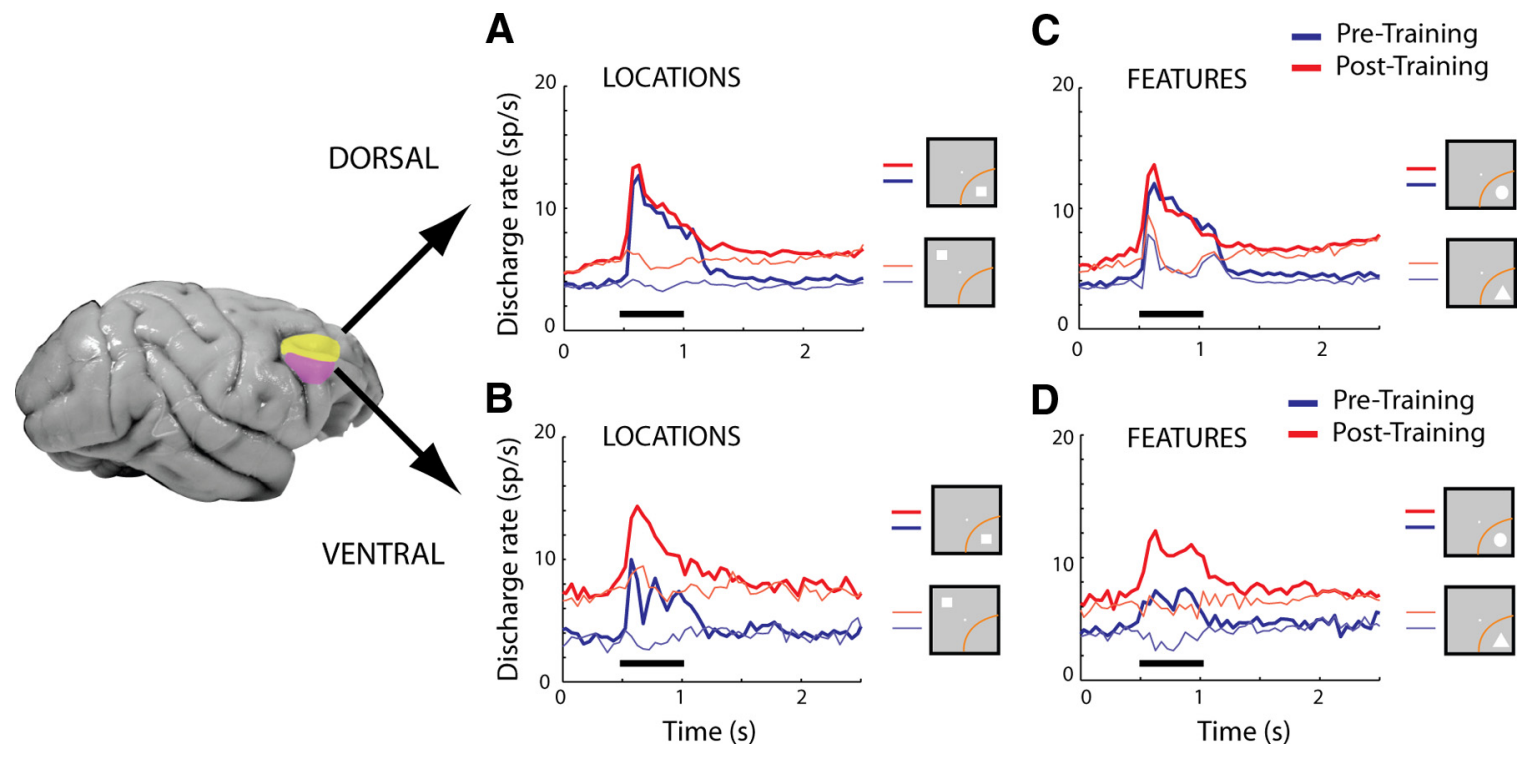

Figure 8. $\quad \boldsymbol{A}-\boldsymbol{D}$, Population peristimulus time histograms averaging visually responsive neurons recorded from each prefrontal region before training and after training in all working memory tasks. Conventions are the same as in Figure 6.
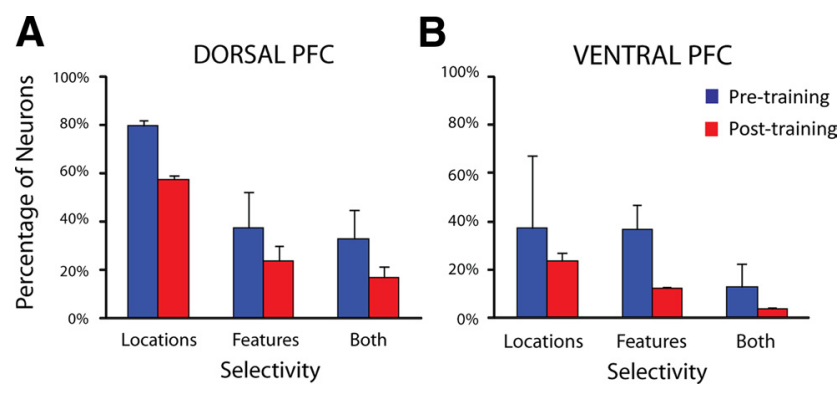

Figure 9. $A, B$, Percentage of visually responsive neurons from the dorsal prefrontal cortex (PFC) $(\boldsymbol{A})$ and the ventral PFC $(\boldsymbol{B})$ with significant selectivity (ANOVA test, $p<0.05$ ) for spatial stimuli, feature stimuli, and both. Data from the same monkeys tested before any training and after training to perform the conjunction task are compared.

cortex after training rendered the difference between dorsal and ventral areas nonsignificant $(t$ test, $p>0.05)$. As was the case after spatial training alone, the decrease in stimulus selectivity was the result of an increase in baseline responses that eroded the difference between best and worst responses (Fig. 8).

This analysis evaluated responses to the spatial and feature sets (as were the results before training, providing a direct comparison). Although the monkeys had already been trained to perform the conjunction task at this stage, it could be argued that presentation of the spatial and feature sets alone did not engage working memory for both features and locations. To ensure that the regional differences that continued to be present after training were not due to the demands of the tasks that we used to evaluate selectivity, we analyzed neuronal selectivity in the conjunction task and compared it with that in the feature and spatial tasks. Each conjunction set relied on combinations of two features and two spatial locations that could be presented as cues and could be followed by a match stimulus or a non-match stimulus. We first determined the responses to the best and worst cue locations in the conjunction set and computed a contrast ratio similar to our selectivity index, $(\operatorname{Max}-\operatorname{Min}) /(\operatorname{Max}+\operatorname{Min})$. We then recalculated the same ratio in the spatial set, comparing responses at the same spatial locations as those used in the conjunction set (even if other locations in the spatial set elicited strong or weaker re-

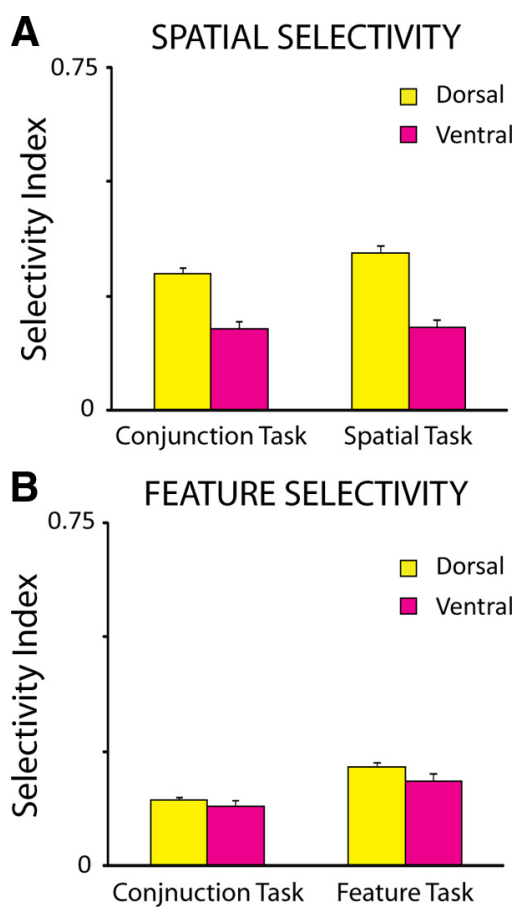

Figure 10. Selectivity index values computed based on the conjunction task after training $A$, Average value of spatial selectivity contrast ratio $(\operatorname{Max}-\operatorname{Min}) /(\operatorname{Max}+\operatorname{Min})$ for the best and worst spatial location tested in the conjunction task for each neuron when these locations were tested as part of the spatial set (left) and as part of the conjunction set (right). B, Average value of shape selectivity contrast ratio for the best and worst feature tested in the conjunction task.

sponses). Results are shown in Figure 10A. A two-way ANOVA confirmed a significant effect of region $\left(p<10^{-5}\right)$ but no significant effect of task and no significant interaction. This result indicates that when performing a behavioral task that requires working memory for a conjunction of spatial locations and features, responses in the ventral and dorsal prefrontal cortexes remain significantly different in terms of their spatial selectivity.

Our analysis also revealed no significant difference in terms of shape selectivity between areas after training, which was the result 


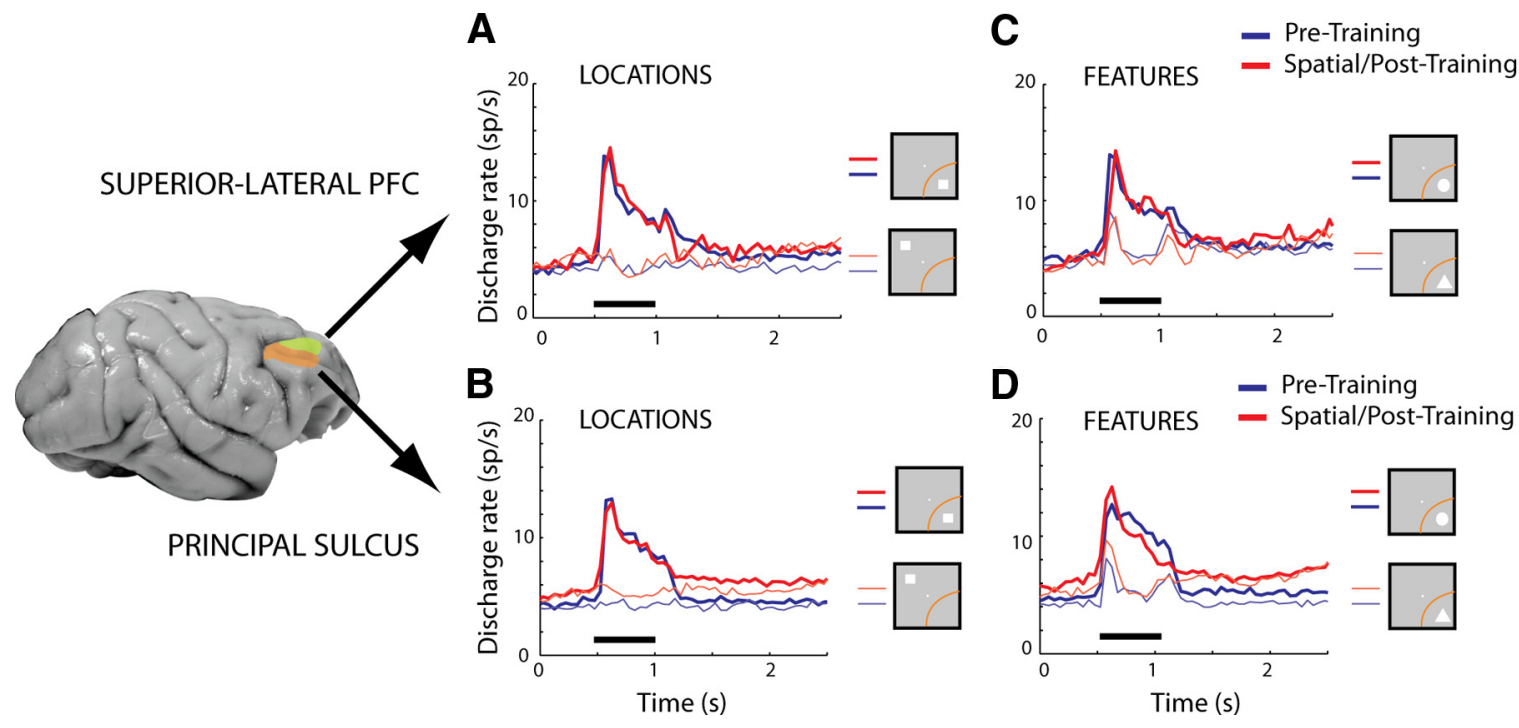

Figure 11. $\quad \boldsymbol{A}-\boldsymbol{D}$, Population peristimulus time histograms averaging visually responsive neurons from two parts of the dorsal prefrontal cortex: the superior-lateral prefrontal cortex $(\mathrm{PFC})(\boldsymbol{A}, \boldsymbol{C})$, and the principal sulcus area $(\boldsymbol{B}, \boldsymbol{D})$. Responses are averaged from neurons recorded before training and after training in either of the working memory tasks. Conventions are the same as in Figure 6.

of decreased selectivity in the ventral prefrontal cortex (Fig. 5C). Analysis of responses from the conjunction task in the same fashion confirmed this finding (Fig. 10B). A further decrease in the average selectivity index for features was observed in the conjunction task compared to the feature task, enhancing the effect of training.

\section{Delay responses}

After training, we found a significant increase of neurons with delay period activity across the prefrontal cortex (comparing the same animals before any training and after training in all tasks): 23 versus $41 \%$ in the dorsal prefrontal cortex and 13 versus 32\% in the ventral prefrontal cortex. The increase was significant for both areas ( $\chi^{2}$ test, $\left.p<0.005\right)$. Firing rate in the delay period also increased, particularly in the ventral prefrontal cortex (Fig. 8).

Contrary to the predictions of the Integrative model, regional differences persisted in terms of stimulus selectivity even when we based our analysis on the delay period. We observed a higher percentage of dorsal (44\%) than ventral prefrontal neurons (11\%) with spatial selectivity in the delay period and a greater magnitude of spatial selectivity among neurons with delay period activity (mean SI $=0.45$ vs $0.32, t$ test, $p<0.05$ ). Comparable percentages of neurons in the two areas were selective for shapes (17 vs 10\%). In terms of neurons selective for both locations and shapes, we saw no difference compared to the pretraining stage (7\% pre, $7 \%$ post, pooled from both prefrontal subdivisions).

\section{Specialization within the dorsal prefrontal cortex}

A recent study demonstrated that lesions restricted to the principal sulcus region and to the region of the dorsal prefrontal cortex superior to it (superior-lateral prefrontal cortex) differentially affect performance in a working memory task (Buckley et al., 2009). In particular, lesions of the superior-lateral region did not impair performance of the task, which required selection of a stimulus depending on different attributes (shape or color) of a cue. This finding prompted us to examine the properties of neurons in the principle sulcus and superior-lateral regions separately. We therefore analyzed the responses of 500 principal sulcus and 276 superior-lateral prefrontal neurons in three monkeys.

Before training, functional properties of the two regions were virtually identical. Similar percentages of neurons were activated by stimulus presentation in the principal sulcus and superiorlateral prefrontal cortex (39 and 33\% respectively, vs $9 \%$ for the ventral prefrontal cortex). Also, similar percentages of neurons were selective for spatial locations (72\% principal sulcus and 65\% of superior-lateral neurons vs $25 \%$ for the ventral prefrontal cortex) and for shapes (38\% principal sulcus and 29\% superiorlateral neurons vs $26 \%$ for the ventral prefrontal cortex). The principal sulcus region exhibited a higher magnitude of spatial selectivity than the superior lateral prefrontal cortex $(0.66$ and 0.59 vs 0.55 for the ventral prefrontal cortex), whereas shape selectivity was lowest for the superior-lateral cortex ( 0.44 and 0.35 vs 0.49 for the ventral prefrontal cortex).

After training in any of the tasks, we examined responses of 700 principal sulcus and 146 superior-lateral prefrontal neurons of two monkeys. We found that the increase in firing rate and decrease in stimulus selectivity that we reported for the dorsal prefrontal cortex as a whole was localized in the principal sulcus region. The average firing rate of superior-lateral neurons remained essentially unchanged (Fig. $11 A-C$ ). We also saw no decline in either spatial or shape selectivity among superior-lateral neurons (mean SI $=0.59$ pretraining vs 0.62 post-training for spatial and 0.34 vs 0.41 for feature stimuli).

\section{Discussion}

Our study examined the functional organization of the prefrontal cortex and how it is affected by learning to perform tasks that require working memory for the spatial locations and features of stimuli. Monkeys were also trained to remember both the location and features of the stimulus to perform the conjunction working memory task. To our knowledge, this is the first study employing a longitudinal design to examine the effects of training on the functional properties of cortical areas at different stages of training.

The nature of information represented in the discharges of prefrontal neurons has been a matter of debate. Anatomical projections from the posterior parietal cortex, the end stage of the dorsal visual pathway, project mostly to the dorsal prefrontal cortex (areas 8 and 46), whereas projections from the inferior temporal cortex and ventral visual stream innervate areas 12 and 45 of the ventral prefrontal cortex (Petrides and Pandya, 1984; 
Selemon and Goldman-Rakic, 1988; Cavada and GoldmanRakic, 1989; Constantinidis and Procyk, 2004). This relative segregation of anatomical inputs led to the postulate that the prefrontal cortex is organized in a domain-specific fashion, with spatial information predominantly represented in the dorsal prefrontal cortex and feature information in the ventral prefrontal cortex (Wilson et al., 1993). These conclusions were challenged by subsequent studies, suggesting that dorsal prefrontal neurons exhibit significant shape selectivity and ventral neurons exhibit spatial selectivity, particularly after monkeys have been trained in tasks that required them to remember both the location and identify of a stimulus (Rao et al., 1997; Rainer et al., 1998). This integrative theory postulates that the prefrontal cortex, because of its intrinsic organization that places parietal and temporal inputs in relative proximity to each other, has the capacity to integrate spatial and feature information for the needs of cognitive tasks and that functional differentiation is primarily the effect of training.

Our recordings performed in animals naive to working memory tasks revealed significant differences between the dorsal and ventral prefrontal cortexes in terms of the two regions' responsiveness to stimuli, their selectivity for spatial and nonspatial information, and the effects of training. Training did alter the functional properties of neurons in both the dorsal and ventral subdivisions, although significant functional differences between the areas remained.

The results we report are consistent with some aspects of the integrative theory, as we observed neurons throughout the prefrontal cortex selective for both locations and shapes. However, such integrative neurons were also present in naive monkeys and did not become more frequent as a result of training. Although these animals were not explicitly trained to perform an integrative task, it appears that the demands of everyday experience necessitate the integration of information in the prefrontal cortex. In sum, our experiments rule out some of the basic premises of the integrative theory, although the domain-specific organization is not absolute either.

\section{Dorsal versus ventral differences}

Dorsal and ventral prefrontal cortexes exhibited a number of functional differences in terms of their stimulus selectivity in our experiments. Dorsal prefrontal cortex consistently exhibited a higher percentage of neurons selective for spatial location (Fig. 9) and a higher magnitude of spatial selectivity (Fig. 5). This result appears consistent with a domain-specific organization, although we should emphasize that the difference was quantitative rather than qualitative; ventral prefrontal neurons did exhibit spatial selectivity as well (Fig. 9B). In fact, the presence of spatial selectivity in the ventral prefrontal cortex has been offered as evidence in support of the integrative model of the prefrontal cortex (Rao et al., 1997; Rainer et al., 1998). We should also point out that selectivity for spatial locations has since been described in the inferior temporal cortex (Op De Beeck and Vogels, 2000; DiCarlo and Maunsell, 2003), suggesting that the presence of such selectivity in the ventral prefrontal cortex reflects the information already present in the ventral visual stream. This conclusion is further strengthened by the fact that spatial selectivity in the ventral prefrontal cortex declined after training in tasks that required spatial information or a combination of spatial and feature information.

Ventral prefrontal neurons were generally less likely to be driven by any stimulus in our set, particularly before training. This finding is consistent with a higher specialization for stimulus features such as that reported for inferior temporal neurons (Gross et al., 1972; Desimone et al., 1984; Tanaka et al., 1991; Fujita et al., 1992). However, selectivity for the feature set we used was less distinguishing of the two areas. Although the magnitude of shape selectivity was higher for ventral prefrontal neurons before training (Fig. 3A), the percentages of selective neurons and SI values at other training intervals were not found to be significantly different between the two areas. These results were not unexpected, as we made use of a limited stimulus set that could not test for very narrow shape selectivity as that described previously for faces and complex objects in the ventral prefrontal cortex (Ó Scalaidhe et al., 1997, 1999). The shape selectivity of the dorsal prefrontal cortex that we report here is similar to the selectivity for geometric shapes reported for the posterior parietal cortex and the frontal eye fields (Sereno and Maunsell, 1998; Janssen et al., 2008; Peng et al., 2008). In this instance too, the shape selectivity of the dorsal prefrontal region appears to be accounted for by selectivity in afferent areas, rather than being a unique property or brought about by training in a conjunction task.

Human imaging studies have also been equivocal about the functional organization of the prefrontal cortex. Some human imaging lent support to the idea that specialized processing occurs within the two prefrontal subdivisions (Adcock et al., 2000; Leung et al., 2002; Sala and Courtney, 2007; Volle et al., 2008), although differences between the two areas have also been described in terms of cognitive operations rather than type of information (Owen et al., 1996, 1998; Stern et al., 2000). We should point out that our recordings were limited to the posterior half of the lateral prefrontal cortex; by some accounts more abstract information is represented in the anterior half (Badre and D'Esposito, 2009), and we cannot rule out that these parts of the lateral prefrontal cortex are less tied to stimulus properties.

\section{Nature of training effects}

Training on the working memory tasks led to recruitment of more neurons, particularly in the ventral prefrontal cortex and during the delay interval. The recruitment of a greater proportion of ventral neurons after training points to a greater reliance on the ventral prefrontal cortex for the mastery of the task and gives credence to the idea that the prefrontal cortex is organized in a process-specific manner as well. Lesions studies also confirm that the ventral prefrontal cortex is particularly critical for learning to perform new tasks (Buckley et al., 2009).

Differential effects of training were also found when we distinguished between two subdivisions of the dorsal prefrontal cortex, the principal sulcus and the superior-lateral prefrontal cortex. Although these two areas were very similar in terms of their stimulus selectivity, the increase in firing rate among dorsal prefrontal neurons in our experiments was localized in the zone around the principal sulcus (Fig. 11). Decreases in stimulus selectivity were also specific for the dorsal region. This result is also consistent with lesion studies, suggesting that the principal sulcus region in particular is critical for maintenance in memory of task-related information, whereas lesion of the superior lateral prefrontal cortex produces essentially no deficit in that respect (Buckley et al., 2009).

\section{References}

Adcock RA, Constable RT, Gore JC, Goldman-Rakic PS (2000) Functional neuroanatomy of executive processes involved in dual-task performance. Proc Natl Acad Sci U S A 97:3567-3572.

Asaad WF, Rainer G, Miller EK (1998) Neural activity in the primate prefrontal cortex during associative learning. Neuron 21:1399-1407. 
Baddeley A (2003) Working memory: looking back and looking forward. Nat Rev Neurosci 4:829-839.

Badre D, D'Esposito M (2009) Is the rostro-caudal axis of the frontal lobe hierarchical? Nat Rev Neurosci 10:659-669.

Brainard DH (1997) The Psychophysics Toolbox. Spat Vis 10:433-436.

Buckley MJ, Mansouri FA, Hoda H, Mahboubi M, Browning PG, Kwok SC, Phillips A, Tanaka K (2009) Dissociable components of rule-guided behavior depend on distinct medial and prefrontal regions. Science 325: $52-58$.

Cavada C, Goldman-Rakic PS (1989) Posterior parietal cortex in rhesus monkey: II. Evidence for segregated corticocortical networks linking sensory and limbic areas with the frontal lobe. J Comp Neurol 287:422-445.

Compte A, Constantinidis C, Tegner J, Raghavachari S, Goldman-Rakic PS, Wang X-J (2001) Spectral analysis and burstiness of prefrontal cortex single unit activity during a spatial working memory task. Soc Neurosci Abstr 27:852-853.

Constantinidis C, Procyk E (2004) The primate working memory networks. Cogn Affect Behav Neurosci 4:444-465.

Constantinidis C, Franowicz MN, Goldman-Rakic PS (2001) The sensory nature of mnemonic representation in the primate prefrontal cortex. Nat Neurosci 4:311-316.

Desimone R, Albright TD, Gross CG, Bruce C (1984) Stimulus-selective properties of inferior temporal neurons in the macaque. J Neurosci 4: 2051-2062.

DiCarlo JJ, Maunsell JH (2003) Anterior inferotemporal neurons of monkeys engaged in object recognition can be highly sensitive to object retinal position. J Neurophysiol 89:3264-3278.

Felleman DJ, Van Essen DC (1991) Distributed hierarchical processing in the primate cerebral cortex. Cereb Cortex 1:1-47.

Fujita I, Tanaka K, Ito M, Cheng K (1992) Columns for visual features of objects in monkey inferotemporal cortex. Nature 360:343-346.

Funahashi S, Bruce CJ, Goldman-Rakic PS (1989) Mnemonic coding of visual space in the monkey's dorsolateral prefrontal cortex. J Neurophysiol 61:331-349.

Fuster JM (1973) Unit activity in prefrontal cortex during delayed-response performance: neuronal correlates of transient memory. J Neurophysiol 36:61-78.

Goldman-Rakic PS (1987) Circuitry of the prefrontal cortex and the regulation of behavior by representational knowledge. In: Handbook of Physiology (Plum F, Mountcastle VB, eds), pp 373-417. Bethesda, MD: American Physiological Society.

Goldman-Rakic PS (1996) Regional and cellular fractionation of working memory. Proc Natl Acad Sci U S A 93:13473-13480.

Gross CG, Rocha-Miranda CE, Bender DB (1972) Visual properties of neurons in inferotemporal cortex of the Macaque. J Neurophysiol 35:96-111.

Gross CG, Bender DB, Gerstein GL (1979) Activity of inferior temporal neurons in behaving monkeys. Neuropsychologia 17:215-229.

Harris KD, Henze DA, Csicsvari J, Hirase H, Buzsáki G (2000) Accuracy of tetrode spike separation as determined by simultaneous intracellular and extracellular measurements. J Neurophysiol 84:401-414.

Janssen P, Srivastava S, Ombelet S, Orban GA (2008) Coding of shape and position in macaque lateral intraparietal area. J Neurosci 28:6679-6690.

Leung HC, Gore JC, Goldman-Rakic PS (2002) Sustained mnemonic response in the human middle frontal gyrus during on-line storage of spatial memoranda. J Cogn Neurosci 14:659-671.

Li W, Piëch V, Gilbert CD (2004) Perceptual learning and top-down influences in primary visual cortex. Nat Neurosci 7:651-657.

Meyer T, Constantinidis C (2005) A software solution for the control of visual behavioral experimentation. J Neurosci Methods 142:27-34.

Meyer T, Qi XL, Constantinidis C (2007) Persistent discharges in the prefrontal cortex of monkeys naive to working memory tasks. Cereb Cortex 17 [Suppl] 1:i70-i76.

Miller EK (2000) The prefrontal cortex and cognitive control. Nat Rev Neurosci 1:59-65.

Miller EK, Cohen JD (2001) An integrative theory of prefrontal cortex function. Annu Rev Neurosci 24:167-202.

Op De Beeck H, Vogels R (2000) Spatial sensitivity of macaque inferior temporal neurons. J Comp Neurol 426:505-518.
Ó Scalaidhe SP, Wilson FA, Goldman-Rakic PS (1999) Face-selective neurons during passive viewing and working memory performance of rhesus monkeys: evidence for intrinsic specialization of neuronal coding. Cereb Cortex 9:459-475.

Ó Scalaidhe S, Wilson FA, Goldman-Rakic PS (1997) Areal segregation of face-processing neurons in prefrontal cortex. Science 278:1135-1138.

Owen AM, Doyon J, Petrides M, Evans AC (1996) Planning and spatial working memory: a positron emission tomography study in humans. Eur J Neurosci 8:353-364.

Owen AM, Stern CE, Look RB, Tracey I, Rosen BR, Petrides M (1998) Functional organization of spatial and nonspatial working memory processing within the human lateral frontal cortex. Proc Natl Acad Sci U S A 95:7721-7726.

Pelli DG (1997) The VideoToolbox software for visual psychophysics: transforming numbers into movies. Spat Vis 10:437-442.

Peng X, Sereno ME, Silva AK, Lehky SR, Sereno AB (2008) Shape selectivity in primate frontal eye field. J Neurophysiol 100:796-814.

Petrides M, Pandya DN (1984) Projections to the frontal cortex from the posterior parietal region in the rhesus monkey. J Comp Neurol 228: 105-116.

Preuss TM, Goldman-Rakic PS (1991) Architectonics of the parietal and temporal association cortex in the strepsirhine primate Galago compared to the anthropoid primate Macaca. J Comp Neurol 310:475-506.

Rainer G, Asaad WF, Miller EK (1998) Memory fields of neurons in the primate prefrontal cortex. Proc Natl Acad Sci U S A 95:15008-15013.

Rao SC, Rainer G, Miller EK (1997) Integration of what and where in the primate prefrontal cortex. Science 276:821-824.

Recanzone GH, Merzenich MM, Jenkins WM, Grajski KA, Dinse HR (1992) Topographic reorganization of the hand representation in cortical area $3 \mathrm{~b}$ owl monkeys trained in a frequency-discrimination task. J Neurophysiol 67:1031-1056.

Recanzone GH, Schreiner CE, Merzenich MM (1993) Plasticity in the frequency representation of primary auditory cortex following discrimination training in adult owl monkeys. J Neurosci 13:87-103.

Romanski LM, Tian B, Fritz J, Mishkin M, Goldman-Rakic PS, Rauschecker JP (1999) Dual streams of auditory afferents target multiple domains in the primate prefrontal cortex. Nat Neurosci 2:1131-1136.

Sala JB, Courtney SM (2007) Binding of what and where during working memory maintenance. Cortex 43:5-21.

Schwartz EL, Desimone R, Albright TD, Gross CG (1983) Shape recognition and inferior temporal neurons. Proc Natl Acad Sci U S A 80:5776-5778.

Selemon LD, Goldman-Rakic PS (1988) Common cortical and subcortical targets of the dorsolateral prefrontal and posterior parietal cortices in the rhesus monkey: evidence for a distributed neural network subserving spatially guided behavior. J Neurosci 8:4049-4068.

Sereno AB, Maunsell JH (1998) Shape selectivity in primate lateral intraparietal cortex. Nature 395:500-503.

Stern CE, Owen AM, Tracey I, Look RB, Rosen BR, Petrides M (2000) Activity in ventrolateral and mid-dorsolateral prefrontal cortex during nonspatial visual working memory processing: evidence from functional magnetic resonance imaging. Neuroimage 11:392-399.

Tanaka K, Saito H, Fukada Y, Moriya M (1991) Coding visual images of objects in the inferotemporal cortex of the macaque monkey. J Neurophysiol 66:170-189.

Tovee MJ, Rolls ET, Azzopardi P (1994) Translation invariance in the responses to faces of single neurons in the temporal visual cortical areas of the alert macaque. J Neurophysiol 72:1049-1060.

Ungerleider LG, Mishkin M (1982) Two cortical visual systems. In: Analysis of Visual Behavior (Ingle DJ, Goodale MA, Mansfield RJW, eds), pp 549586. Cambridge, MA: MIT.

Volle E, Kinkingnéhun S, Pochon JB, Mondon K, Thiebaut de Schotten M, Seassau M, Duffau H, Samson Y, Dubois B, Levy R (2008) The functional architecture of the left posterior and lateral prefrontal cortex in humans. Cereb Cortex 18:2460-2469.

Wilson FA, Ó Scalaidhe SP, Goldman-Rakic PS (1993) Dissociation of object and spatial processing domains in primate prefrontal cortex. Science 260:1955-1958. 